\title{
THE MODEL OF DATA PRESENTATION WITH FUZZY PORTRAITS FOR PATTERN RECOGNITION
}

\author{
Aleksandra Maksimova \\ Institute of Applied Mathematics and Mechanics of National Academy of Sciences of Ukraine, \\ 74, Roza Luksemburg st., Donetsk, Ukraine \\ maximova.alexandra@mail.ru, \\ http://iamm.ac.donetsk.ua/en/employees/e934/
}

\begin{abstract}
This paper deals with a data presentation model based on fuzzy portraits. The fuzzy portraits are formed by integral characteristics of pattern classes. It is the basis for fuzzy classifier construction. It is determined that further division of some classes of images into clusters increases the quality of pattern recognition algorithm. The main idea of fuzzy clustering for fuzzy portraits creating and problem of adequate fuzzy partition choice is considered. The paper provides the stages of fuzzy production knowledge base construction on the basis of fuzzy portraits. The local validity measure for fuzzy portrait is defined. The problem of identification in chemical and food industries is considered as an application of this approach.
\end{abstract}

Keywords: Pattern recognition, fuzzy logic, clustering, data mining, fuzzy classifier.

\section{INTRODUCTION}

Pattern recognition problems very often include fuzzy aspects. According to Lotfi Zadeh, the transition between classes is more gradual than intermittent. This statement is confirmed by practice of problem solving. In many empirical fields the standard approach that seeks a boundary between several classes gives no satisfactory results. There are different forms of fuzziness, described in [1]. We shall consider the following manifestations of fuzziness: diffusiveness, vagueness and relative position of classes.

In classical pattern recognition problem statement classes of images have to be strongly divided. The separating surface can be a hyperplane or have a rather complicated form. A large group of pattern recognition algorithms is based on estimates computation algorithms (ECO) concept proposed by Yu. I. Zhuravlev [2]. The basic principle of calculating if an object belongs to a class is calculating its closeness to the master object of owner class and farness to the master objects of other classes.

If classes of images have intersections the algorithm for making decisions about whether an object belongs to the class or not plays a big role. Previously, an algorithm based on fuzzy portraits of classes of images was proposed [3]. These fuzzy portraits describe all objects belonging to a certain class of images in general. Fuzzy portraits accumulate the information about elements of the class such as frequency of object popularity, degree of objects "concentration" and number of objects in every class. The suggestion is to use these portraits for representing the results of algorithm in the form of fuzzy sets. This approach to a certain degree solves the problem of ambiguous results in the case of intersecting classes. Information about an object's distance from other classes has a substantially smaller significance in this situation and is used only for adjustment of fuzzy sets for aims of quality improvement. However the internal structure of classes is also taken into account. The classes of images can be further divided into groups of clusters that can have intersections too. Thus initial fuzzy portraits are expanded through additional structuring.

The results of this research were presented at the Pattern Recognition and Image Processing Conference in Belarus, Minsk and partially presented in [4].

\section{FEATURES OF MODIFICATION OF PATTERN RECOGNITION PROBLEM}

The following problem statement will be considered. It is a fuzzy modification of classification problem. Let us suppose that there is a set of training samples 
$Y=\left\{\left(x^{(i)}, v^{(i)}\right) \mid x^{(i)} \in X, v^{(i)} \in V, i=1, \ldots, n\right\}, \quad$ where $x^{(i)} \in X \subset R^{m}$ are vectors in the $\mathrm{n}$-dimensional space of images, $V=\left\{v_{i} \mid i=1, \ldots, k\right\}$ - set of classes of images. Pairs $\left(x^{(i)}, v^{(i)}\right)$ set the correspondence between object $x^{(i)}$ and pattern class $v^{(i)}$. It is necessary to produce the algorithm of identification $a_{Y}(x)=\tilde{y}$. Vector $x \in X$ is the input of the algorithm, $X \supseteq X$ - the set of possible input data that depends on the problem domain. Generally, a fuzzy information vector $\tilde{y}$ describing membership $x$ of pattern classes is obtained.

The training samples set can be incomplete, i.e. it may not contain all classes of images. The classes of images may overlap which is a manifestation of a form of fuzziness - diffusiveness of classes of images. For every object that is an input for the algorithm it is necessary to determine its membership degree in every pattern class.

Let us consider a qualitative attribute of an object called "source". A pair of "sources" can produce a pair of images $\overline{x_{1}}$ and $\overline{x_{2}}: \overline{x_{1}}=\overline{x_{2}}, \overline{x_{1}} \in v_{i}, \overline{x_{2}} \in v_{j}, v_{i} \neq v_{j}$, i.e. patterns with same attribute values belong to different classes in the initial training set. In a situation when this attribute is a part of classification goal some classes will be nonseparable and their intersection can be very large, to a point where one class is absorbed by another. In such cases when the researcher fails to find a new attribute that would allow to divide the classes, the problem becomes unsolvable.

Such problems are common in quality control laboratories. In these cases a "source" is a product manufacturer that needs to be identified. Many problems in chemical and food industries can be reduced to this modification of the pattern recognition problem.

For such training samples a recognition algorithm based on the use of fuzzy portraits was proposed in [3]. The algorithm's output is a fuzzy information vector set $\tilde{y}=\left(M_{1}, \ldots, M_{i}, \ldots, M_{k}\right)$, where $M_{i}$ is object's membership degree for pattern class $V_{i}$, $k$ is number of classes. Algorithm creates a knowledge base of fuzzy productions where every pattern class has its own rule of inference. The fuzzy inference algorithm works with knowledge base.

We consider a modification of the proposed algorithm. The analysis of empirical data often shows that there are subclasses (i.e. clusters) present in pattern classes. That is why it is useful to perform clustering for objects related to class to obtain a more precise fuzzy portrait.

\section{ANALYSIS OF INITIAL SET}

A necessary step in solving problems of pattern recognition is a preliminary analysis of initial data which is often called exploratory analysis of data. The basic aims of this analysis were formulated by J. Tukey:

- maximum penetration of data;

- the basic structures detection;

- selection of the more important attributes;

- discovering deviations and anomalies;

- testing the basic hypotheses;

- the construction of the initial model.

The proposed approach allows efficiently detecting basic structure of classes of images and constructing data presentation model as a result.

The study of initial data structure allowed to choose an algorithm for solving the problem. Generally every pattern recognition algorithm works well with certain data structure. For example, linear classifiers were created for linearly separable classes. The EM-algorithm [5] works with intersecting classes and implies the existence of a hidden variable. There is a number of algorithms covering similar situation that produce the result in the form of a fuzzy set.

In practice there are samples with one or more classes that consist of several clusters. Let us consider a case of strongly intersecting clusters that have pronounced centers (typical points) but have no clear border between them. It is possible, for example, to get this kind of data by changing some parameters of industrial technological process. A number of attributes changes which creates new clusters. It is reasonable to use fuzzy clustering approach $[1,6]$ for intra-class structure analysis.

A criterion for the quality of the partitions on the original set of clusters is the determining parameter. Moreover, a solution that is optimal from a mathematical point of view does not always meet your goals. The current paper deals with one possible strategy of intra-class structure study with clustering methods.

Let a coverage $Y_{S}$ be defined on the set of images $X$, that is a part of training samples set $Y$. Let $P=\left\{P_{1}, \ldots, P_{q}, \ldots, P_{m}\right\}$ be a named set of attributes, where $m$ is the number of attributes. The attributes are defined on sets of values $X_{P_{q}} \subset X$, $P_{q} \in P$. Similarly to the class $V_{j}$, let us denote the set of images of training samples corresponding to this class as $V_{j}=\left\{\overline{x_{j}^{\left(i_{j}\right)}} \mid i_{j}=1, \ldots, t_{j}\right\}, j=1, \ldots, k$, 
where $k$ is the number of classes represented in training sample, $t_{j}$ is the number of objects in the class with number $j$, and where $\overline{\left.x_{j}^{\left(i_{j}\right)}\right)}=\left(x_{j 1}^{\left(i_{j}\right)}, \ldots, x_{j q}^{\left(i_{j}\right)}, \ldots, x_{j m}^{\left(i_{j}\right)}\right), q=1, \ldots, m$. So the coverage is $Y_{S}=\left\{V_{1}, \ldots, V_{j}, \ldots, V_{k}\right\}$.

Let the set $D_{j}^{q}=p r_{P_{q}} V_{j}=\left\{x_{j q}^{\left(i_{j}\right)} \mid i_{j}=1, \ldots, t_{j}\right\}$ be a projection of class with number $j$ on attribute $P_{q}$.

The basic principle of discriminant analysis is "the effect of essential multidimensionality". According to this principle it is necessary to take into account the information about all attributes and to solve the problem in the m-dimensional space [7]. There are at least two reasons to avoid multiple dimensions. Firstly, data with small training samples gives us a sparse set of points which most pattern recognition algorithms do not process correctly. In this case a clustering by one or several attributes may be not worse than general clustering. Secondly, there are difficulties in substantiating the selection of distance measure which is used in multidimensional clustering.

Thus, we have two strategies for finding clusters. The first one is to perform clustering in the $\mathrm{m}$ dimensional space taking into account the previously described shortcomings of this approach. The second approach is to perform fuzzy clustering for each projection $D_{j}^{q}, q=\overline{1, m}, j=\overline{1, k}$.

Let us consider the general scheme of data analysis and then details of both strategies of intraclass clustering.

Most commonly fuzzy clustering algorithms produce a fuzzy partition or fuzzy coverage as a result. Let us consider a family of fuzzy sets $R(X)=\left\{A^{l} \mid l=1, \ldots, c\right\}, \quad$ where $A_{l}=\left\{\left(x, \mathcal{M}_{l}(x)\right) \mid \mathcal{M}_{l}(x) \in[0,1], x \in X\right\}$ is fuzzy set and $c$ is the number of clusters described by fuzzy set $A_{l} . R(X)$ is a fuzzy $c$-partition when the following condition is satisfied:

$$
\sum_{l=1}^{c} \mathcal{M}_{l}\left(x_{i}\right)=1, i=1, \ldots, n, M_{l} \in[0,1]
$$

$R(X)$ is a fuzzy coverage when the condition of fuzzy coverage is satisfied:

$$
\sum_{l=1}^{c} \mathcal{M}_{l}\left(x_{i}\right) \geq 1, i=1, \ldots, n, \mathcal{M}_{l} \in[0,1]
$$

The fuzzy clustering procedure is performed for every set $V_{j}, j=1, \ldots, k$ giving a fuzzy partition or a fuzzy coverage $R\left(V_{j}\right)$ as a result. It establishes a correspondence between partition $Y_{S}$ and set of fuzzy partitions or coverages $R_{S}=\left\{R_{j}(X) \mid j=1, \ldots, k\right\}$.

The result of such analysis of training sample is the transformed data that is used in the algorithm and it is described by the following data model:

$$
M_{S}=\left\{(V, R(V)) \mid V \in Y_{S}, R \in R_{S}\right\} .
$$

Thus we receive special data structure where a fuzzy coverage corresponds to every set $V \in Y_{S}$.

The major stages of data presentation model creating are the following:

1. The initial learning set $Y$ is prepared based on real data from applied problem. The set of attributes $P$ is defined for current problem.

2. The set of classes of images $V$ is defined and initial set $Y_{S}$ is constructed.

3. The intra-class clustering is carried out for all classes of images and fuzzy partition $R_{s}$ is constructed.

4. The model of data presentation model with fuzzy portraits $M_{S}$ is constructed on the basis of $Y_{S}$ and $R_{S}$.

5. The quality of model $M_{S}$ is tested. If the quality of the model $M_{S}$ does not satisfy the purposes of pattern recognition, then the adjustment of the model by machine learning methods is carried out.

It should be noted that the number of clusters for every class of images $V_{j}$ is unknown in this situation and it is the reason for using unsupervised clustering algorithms [8]. The quality estimation for the fuzzy partition or the fuzzy coverage $R\left(V_{j}\right)$ is carried out from the pattern recognition point of view. Thus we have to use clustering algorithms with super goal.

The described approach gives as a result an integral description of class of images that takes intra-class structure into account. The presence of the number of clusters in a class may be due to technological process specifics. For example, it can be a material change in the composition of substance in the manufacturing process that does not impact the stated quality but generates a homogeneous 
subgroup of images for the class, describing the substance.

\section{DATA REPRESENTATION THROUGH FUZZY PORTRAITS OF CLASSES OF IMAGES AND FUZZY CLASSIFIER GENERATION}

A number of pattern recognition algorithms uses the information about pattern classes structure. In these algorithms the distance function is used to calculate the typical points or build probability distributions of points in classes [9].

It is suggested to present the proposed data structure $M_{S}$ as fuzzy portraits.

Let us introduce certain notions before giving the definition of a fuzzy portrait. Let the quintuple of objects $\left\{\operatorname{name}\left(P_{q}\right), T_{q}, X_{P_{q}}, G, M\right\}$ be called the linguistic variable $L_{q}$ by the attribute $P_{q}$, where name $\left(P_{q}\right)$ denotes the name of linguistic variable,

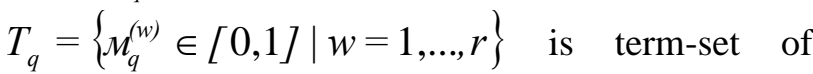
linguistic variable values, $w$ is the cluster number, $r$ is the total number of clusters for all pattern classes. Syntactical rule $G$ that generates variable names is trivial in this case, because all terms are atomic and it simply gives terms the name of a class or a subclass. Semantic rule $M$ is represented as an algorithm for forming term-set membership functions.

Let the fuzzy portrait $S_{j}$ of class of images $V_{j}$ be the fuzzy area in m-dimensional space that is described by the three objects (L,Rule,F) where $L$ is the set of linguistic variables $\left\{L_{q} \mid q=1, m\right\}$, $m$ is the number of attributes, $T_{q}=\left\{\mu_{q}^{i}(x) \mid i=1, l\right\}$ is the set of terms, where $l$ corresponds to number of clusters $R_{j}$ to current class $V_{j}, R$ is set of rules and $F=\left\{F_{A N D}, F_{O R}\right\}$ are the pair of operations AND and OR correspondingly:

$$
\left(\left\{L_{q} \mid q=1, \ldots, m\right\},\left\{\text { Rule }_{q} \mid q=1, \ldots, l\right\},\left\{F_{A N D}, F_{O R}\right\}\right) .
$$

The inference rule Rule $_{q}$ is the following:

$$
\text { IF } \mu_{q}^{1}(x) \text { is } A_{q} \text { AND ...AND } \mu_{q}^{m}(x) \text { is } A_{q} \text { THAN } V_{j} \text {, }
$$

where $A_{q}$ is the defined by $R\left(V_{j}\right)$ cluster. The number of such inference rules corresponds to the number of clusters detected by cluster analysis and can be different for different classes of images.

Let an operator $F_{O R}$ be the function of taking the maximum then fuzzy portrait $S_{j}$ can be determined by function:

$$
F_{S_{j}}=\max _{i=1}^{l}\left(F_{A N D}\left(M_{i}^{1}\left(x^{(j)}\right), \ldots, M_{i}^{m}\left(x^{(j)}\right)\right)\right.
$$

The value of $F_{S_{j}}$ is always in $[0,1]$ and if $F_{S_{j}}(\bar{x})=1$ then $\bar{x}$ belongs to a class of images $V_{j}$ and if $F_{S_{j}}(\bar{x})=0$ then it does not.

Fig. 1 shows an example of fuzzy portrait visualization for class of images in two-dimensional space with two attributes $P_{1}$ and $P_{2}$. The points of initial set for this class are shown on the picture. Bright areas correspond to large membership degrees and white areas represent $F_{S_{j}}(\bar{x})=1$. The areas of fuzzy portrait with less intensity represent class members with admixtures: $0<F_{S_{j}}(\bar{x})<1$.

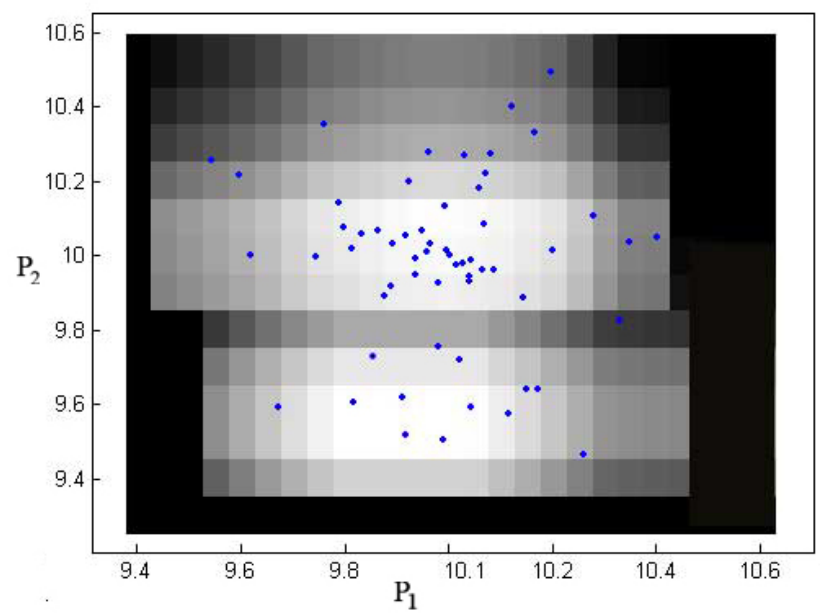

Fig.1 - Example of fuzzy portrait

Let the degree of fuzzy portrait $S_{j}$ be the number of clusters that the class $V_{j}$ consists of. Fig. 1 shows the second degree fuzzy portrait.

Let us denote the local validity measure for fuzzy portraits with:

$$
F_{V_{j}}=\frac{1}{t_{j}} \sum_{i=1}^{t_{j}} F_{S_{j}}\left(\bar{x}_{i}\right)
$$

where $t_{j}$ is the number of initial set elements of class $V_{j}$. This measure estimates the measure of correspondence of the fuzzy portrait to initial set. The maximum value for this measure is one. It is an 
ideal situation that is never reached on real data taking into account the presence of noise.

The choice of operation $F_{A N D}$ and membership function for linguistic variables greatly influence the form of fuzzy portrait area. Some examples of operation AND are given in Table 1.

Table 1. -Function for AND operation

\begin{tabular}{|c|c|l|}
\hline$F_{1}$ & $F(\bar{x})=M_{1}(x) \cdot \ldots \cdot M_{m}(x)$ & $(8)$ \\
\hline$F_{3}$ & $F(\bar{x})=\min \left(M_{1}(x), \ldots, M_{m}(x)\right)$ & $(9)$ \\
\hline$F_{4}$ & $F\left(x_{1}, x_{2}\right)=\max \left(M_{1}(x)+M_{2}(x)-1,0\right)$ & $(10)$ \\
\hline
\end{tabular}

In [3] the following function has been proposed:

$$
F_{2}\left(x_{1}, x_{2}, \ldots, x_{m}\right)=\log _{2}\left(\left(x_{1}+1\right) \cdot \ldots \cdot\left(x_{m}+1\right)\right) / m
$$

where $x_{i} \in[0,1], F_{2}(\bar{x}) \in[0,1]$.

The group of fuzzy inference rules describing fuzzy portraits represents a fuzzy classifier. This type of classifiers was used for pattern recognition purposes recently [10,11]. The fuzzy classifiers produce a fuzzy information vector $\tilde{y}$ as a result. The hardering procedure is used to get crisp answer. The class with maximum membership value is chosen.

The fuzzy classifiers are applied to pattern recognition and data analysis problems and each fuzzy inference rule has linguistic interpretation. That is why the mechanism of knowledge base generation can be used as an instrument for data analysis.

The advantage of this approach is having a meaningful interpretation of the result which is not the case with neural networks, for example.

\section{INTRA-CLASS CLUSTERING BASED ON BATCH POINT-PROTOTYPE APPROACH}

A popular approach for fuzzy clustering is fuzzy c-means model from batch point-prototype clustering models [6, p 16.] that aims to minimize the following functional:

$$
J_{m}(A, C)=\sum_{i=1}^{l} \sum_{k=1}^{n} \mu_{i k}^{m} L_{i k}^{2},
$$

where $A$ is a fuzzy partition like (1), $C=\left(c_{1}, \ldots, c_{l}\right)$ is a set of points prototypes, $m \geq 1$ is a degree of fuzzification, $L_{i k}=\left\|x_{k}-c_{i}\right\| \square$ is a distance.
The output of fuzzy c-means algorithm (fcm) is fuzzy c-partition like (1). Let us call this algorithm fcm-classic.

Let us call the following variation of this algorithm an fcm-projection algorithm. The attribute $P_{q}$ is fixed and fcm is performed on $D_{j}^{q}=p r_{P_{q}} V_{j}$ with the output $R(X)=\left\{A^{l} \mid l=1, \ldots, c\right\}$, where $A_{l}$ is a vector determining membership for every element from $D_{j}^{q}$ to cluster $l$. Multi-dimensional cluster is then reconstructed.

Since fcm works with a fixed number of clusters, which is unknown, it is proposed to make fuzzy portraits of different degrees and choose the best one by the criterion (7). For the same value of criterion for different number of clusters the fuzzy portrait with smaller degree is selected.

The major stages of fuzzy portraits generation algorithm for class of images $V_{j}$ is given below. The parameter $\mathrm{C}$ of algorithm is the current degree of fuzzy portrait.

1. $\mathrm{C}=1$ is fixed and fuzzy portrait of the first degree is made with fcm-classic algorithm. $F_{V_{j}}^{1,0}$ is calculated by (7).

2. The degree of fuzzy portrait $\mathrm{C}$ is incremented by one point.

3. The fuzzy portraits of C-degree are made for every $D_{q}^{j}, q=1, \ldots, m . F_{s_{j}}^{c q}$ is calculated by (7).

4. The best fuzzy portrait is chosen:

$$
F_{s_{j}}^{c}=\max _{q=0}\left(F_{S_{j}}^{c q}\right) .
$$

5. The dynamic of $F_{s_{j}}^{c}$ changes with $\mathrm{C}$ increasing is estimated. If the quality of fuzzy portraits is decreasing with $\mathrm{C}$ increasing then the algorithm is stopped, else steps 3 to 5 are repeated.

It is necessary to use a modification of sliding method [12] to create the membership function that takes weights of initial set elements from c-partition $R_{j}$ into account.

\section{ILLUSTRATIVE EXAMPLE}

Let us consider the algorithm's performance on wine classification problem from UCI repository [13]. We have to solve problem of wine identification on the basis of chemical analysis data (like alcohol, malic acid, nonflavonoid phenol concentration, color intensity and other). It is a 
known set of data of chemical analysis of wines grown in the same region in Italy but derived from three different cultivars.

The problem using the approach described in part 5 of this paper based on fcm approach.

Fuzzy portraits of degrees 1 through 4 were created with different types of operations $F_{A N D}$ : $F_{1}, F_{2}, F_{3}, F_{4}$. Table 2 shows values for $F_{V_{1}}$ for different types of $F_{A N D}$ and different number of clusters created by fcm-classic.

Table 2. - Class $V_{1}$ (m-dimensional clustering)

\begin{tabular}{|c|c|c|c|c|}
\hline clusters & $F_{2}$ & $F_{1}$ & $F_{3}$ & $F_{4}$ \\
\hline 1 & 0.79 & 0.58 & 0.65 & 0.54 \\
\hline 2 & $\mathbf{0 . 8 4}$ & 0.67 & 0.72 & 0.65 \\
\hline 3 & 0.82 & 0.62 & 0.67 & 0.60 \\
\hline 4 & 0.69 & 0.43 & 0.47 & 0.39 \\
\hline
\end{tabular}

Table 3 shows values for $F_{V_{1}}$ for different types of $F_{A N D}$ and different number of clusters created by fcm-projection algorithm.

Table 3. - Class $V_{1}$ - (clustering by projection)

\begin{tabular}{|c|l|l|}
\hline Clusters & $F_{2}$ & $F_{1}$ \\
\hline 1 & -- & -- \\
\hline 2 & $0.84 ; \mathbf{0 . 8 5}$ & $0.65 ; 0.68$ \\
\hline 3 & $0.8 ; 0.82$ & $0.61 ; 0.63$ \\
\hline 4 & $0.7 ; 8.1$ & $0.37 ; 0.62$ \\
\hline
\end{tabular}

From experiment results the best fuzzy portraits with maximum of local validity measure were selected.

Moreover, it was ascertained that quality of pattern recognition depends directly on the fuzzy portraits quality calculated by (7). It was demonstrated that clustering by projection increased the quality of pattern recognition and decreased computational complexity for this particular type of problems where clustering is due to variations in the technological process, i.e. to the change only in some attributes.

The algorithm without clustering proposed in produced about $8 \%$ of errors on the model data with distinct interpretation of result. After clustering of every class of training sample two clusters inside the first class were obtained and the modified algorithm improved the recognition rate to $96 \%$.

\section{THE AREA OF APPLICATION FOR THE CURRENT APPROACH. REMARKS AND RECOMMENDATIONS}

The main idea of current approach is finding qualitative fuzzy portraits that give integral characteristic of a class of images. This approach allows to make fuzzy classifiers with great generalization ability. This approach is used for problems of identification and control of quality in chemistry and foods industries. Results of organoleptic analysis and data from chemistry devices are used as attributes. Such problems often have the property of a priori inseparability and large noisiness.

To improve pattern recognition quality we have to use different types of fuzzy clustering algorithms, make extra transformation of initial set and find bad points. [8] uses clustering algorithms to find bad points.

In pattern recognition problems it is often sufficient to have the answer: "This image does not belong to this class". In such cases it is enough to use only those rules from the knowledge base that relate to particular classes to receive the necessary answer. This approach is important for the situation when the system can not answer the question "What class does this image belong to?” either because of the intersection of several classes, or, in a situation when this class of images is not described in the knowledge base.

In addition, we present an algorithm of fuzzy portraits creation on the basis of possibilistic clustering D-AFC(c) algorithm which has the following major stages.

1. Fuzzy portraits for every class of training sample are created without clustering. A semantic rule based on the frequency of object occurrence for every attribute is used for term-set forming [14].

2. The possibilistic clustering of pattern classes is conducted using D-AFC(c) algorithm of fuzzy clustering for every attribute $q$ which produces fuzzy coverage $R_{j}^{q}(X)$ for every class $j$. The best fuzzy coverage $R_{j}(X)$ is determined through solving the equation $R_{j}(X)=\arg \max _{\forall R_{j}^{q}(X)} F\left(R_{j}^{q}(X)\right)$, where $F\left(R_{j}^{q}(X)\right)$ is the criterion of fuzzy allotment quality:

$$
F\left(R_{j}^{q}(X)\right)=\sum_{w=1}^{c_{j}^{q}} \frac{1}{n_{w}} \sum_{i=1}^{n} \mu_{w}^{q}\left(x_{i}\right)-\alpha \cdot c_{j}^{q},
$$


where $n_{w}$ is the number of elements on the fuzzy cluster $w$. The D-AFC(c) algorithm is described in [8].

3. If there are adequate fuzzy coverages they are used to adjust fuzzy portraits.

The fuzzy inference is carried out using the generated knowledge base of fuzzy productions. For every fuzzy portrait $S_{j}$ one fuzzy inference rule is formed in case of no clusters or several fuzzy inference rules for every cluster in case of successful clustering. The linguistic variables values are calculated with fuzzy portraits terms $T_{q}: \mathcal{M}_{q}^{(w)}(\bar{x})=\mathcal{M}_{q}^{(w)}\left(x_{q}\right)$ on fuzzification stage of fuzzy inference algorithm. The aggregation stage is carried out using $F_{2}(6)$.

On it a defuzzification procedure can be carried out to obtain a distinct result if need be.

The discrete fuzzy set $\tilde{y}$ defined on the set of classes of images is the result of fuzzy inference algorithm. On it hardering operation can be carried out to obtain a distinct result if need be.

\section{CONCLUSION}

In conclusion let us note certain features of the proposed algorithm. The main idea of this paper is data presentation model by fuzzy portraits making for pattern recognition problem decision.

This approach is useful for pattern recognition problems with great number of classes. The result of the recognition procedure is given in the form of a fuzzy set which, in many cases, is more natural for the expert, who makes a final decision on whether the pattern belongs to a class and therefore can be easily integrated into decision-making support system.

The independent consideration of individual attributes on different stages of the algorithm allows to avoid preliminary normalization of training samples and introducing distance measure which is usually done when processing multidimensional data. That is why it is proposed to extract main information about dissimilarity of classes component-wise using projection on attributes

The proposed variation of the algorithm using the fuzzy portraits created on the basis of individual attributes can be expanded further through using not only conventional one-dimensional projections, but also two-, three-dimensional etc. projections of original images as basic data subsets. Ideology of fuzzy portraits construction will be preserved. It is quite natural to call the one-dimensional variation of the proposed algorithm a first-order algorithm and the variation that takes into consideration not more than m-dimensional projections a k-order algorithm.
The approach described allows to not only solve machine learning problem but to store the results of data analysis procedure in a knowledge base. This method may be used for automatic context expansion in expert systems [14].

The algorithm gave good results on model data with error rate not more than $2 \%$ (hardering procedure is used). Algorithm was tested on "wine" problem from UCI data repository [15] with the error rate of $4 \%$.

This approach has already been briefly discussed in [4], but the current article provides a more detailed presentation of the proposed concept.

\section{REFERENCES}

[1] D.A. Viattchenin, Fuzzy Methods of Automatic Classification, Technoprint, Minsk, 2004. p. 219 (in Russian)

[2] Yu.I. Zhuravlev, An algebraic approach to the solution of pattern recognition and identification problems, Probl. Kibernet, (33) (1978), pp. 5-68. (in Russian).

[3] V.A. Kozlovskii, A.Ju. Maksimova, Decision of pattern recognition problem with fuzzy portraits of classes, Artificial Intelligence, (4) (2010), pp. 221-228. (in Russian)

[4] V.A. Kozlovskii, A.Yu. Maksimova, Algorithm of pattern recognition with intra-class clustering, Proceedings of 11th International Conference "Pattern Recognition and Information Processing”, Minsk, Belarus 18-20 May 2011, pp. 54-57.

[5] M. Schlesinger, V. Hlavac, Ten Lectures on Statistical and Structural Pattern Recognition, Springer, 2002, p. 544.

[6] J.C. Bezdek, J.M. Keller, R. Krishnapuram, N.R. Pal, Fuzzy Models and Algorithms for Pattern Recognition and Image Processing, Springer Science, New York, p. 776.

[7] S.A. Aivasian, V.M. Buchstaber, Applied statistics. Classification and reduction of dimensionality, Moscow, 1989, p. 608. (in Russian)

[8] F. Klawonn, R. Kurse and H. Timm, Fuzzy Shell Cluster Analysis. [http://public.fhwolfenbuettel.de/ klawonn/Papers/klawonnetal udine97.pdf]. University of Magdeburg. Magdeburg, Germany. pp. 1-15.

[9] M. Friedman, A. Kandel, Introduction to Pattern Recognition: Statistical, Structural, Neural and Fuzzy Logic Approaches, World Scientific Publishing Company. Singapore. 1999.

[10] H. Ishibuchi, T. Nakashima, M. Nii, Classification and Modeling with Linguistic Information Granules: Advanced Approaches 
to Linguistic Data Mining, Springer, 2004. p. 318.

[11] H. Ishibuchi, K. Nozaki and H. Tanaka, Distributed representation of fuzzy rules and its application to pattern classification, Fuzzy Sets and Systems, (52) (1992), pp. 21-32

[12] V.A. Kozlovskii, A.Yu. Maksimova, Pattern recognition algorithm based on uzzy approach, Artifical Intelligent, (4) (2008), pp. 594-599 (in Russian).

[13] A. Frank, A. Asuncion, UCI Machine Learning Repository [http://archive.ics.uci.edu/ml]. Irvine, CA: University of California, School of Information and Computer Science. 2012.

[14] D.A. Viattchenin, A direct algorithm of possibilistic clustering with partial supervision, Journal of Automation, Mobile Robotics and Intelligent Systems, (3) 1 (2007), pp. 29-38.
[15] A.Yu. Maksimova, O.O. Varlamov, Mivar expert system for pattern recognition on the basis of fuzzy classification and data domains modeling with automatic context expantions, Izvestia YuFU. Technics, (12) 125 (2011), pp. 77-87.

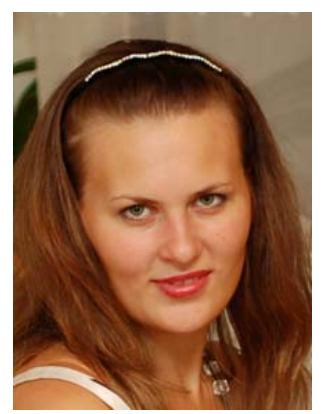

Aleksandra Maksimova, received MSc degree (2004) of Computer Science from Donetsk National University. She is currently a PhD candidate with the Institute of Applied Mathematics and Mechanics. Primary research interests are pattern recognition, data mining, fuzzy clustering. 